\title{
Studies on the TSH in Urine and Blood
}

\section{Osamu ISHIGAMI}

Department of Obsterics and Gynecology, Kobe University School of Medicine, Kobe, Japan

(Director : Professor Yasuo Ueda, M.D.)

As Compared with gonadotropin, the study on the problem of TSH in body fluid needs to be investigated further though it belongs to a similar hormonal group. Some of the reasons for the delay in TSH investigation may be the back of an appropriate procedure for extracting TSH and probably the lack of a precise test for estimation. Its unstable condition compared with gonadotropin as well as the unusual minute quantity in body fluid also presents difficulties. The present study was undertaken to seek a most suitable method for extracting a micro-trace of TSH in urine and blood comparative biochemical studies were than mode. The results of this investigation on the variation of TSH in female body fluid were as follows:

1. Extraction of the Urinary TSH

Although a stability test was made of TSH by direct addition in urine, no evidence of inactivity of TSH in urine could be found. To detect this extremely small amount of TSH in urine, the following methods were tried, namely, Bates' percolation method, four fold alcoholic sedimentation method and Kaolin adsorption method. The best extraction method seemed to be Bates' percolation method from the sediment obtained by means of four-fold alcoholic sedimentation.

2. Comparative Studies on the Biochemical Characters of TSH in Urine and Blood

For comparative biochemical studies, both specimens require special extractions. Hence, Sephadex gel filtration and polyacrylamide gel electrophoresis methods were used.

It was found that the Urinary TSH was more retarded on Sephadex gel filtration than was the TSH in blood and with a larger $\mathrm{Kd}$ volume. While with the method of polyacrylamide gel electrophoresis urinary TSH showed many divided bands compared with blood TSH, these bands, indicative of biological activity, were found to be in the same area though blood TSH displayed a greater width of the band than that of urinary TSH. It was also revealed that specific activity in blood TSH seemed to be higher when compared with urinary TSH. Thus, it is evident that urinary TSH contained lesser molecular weight than the blood TSH which apparently is weak in TSH active principle presumably isolated from blood

3. Relative TSH Values in Urine and Blood

Presence of urinary TSH was demonstrated from a large amount of specimen of urine and blood by employing a most reliable method, and these micro-extracted substances isolated in pure from enabled us to identify their biologic character. Urinary 
and blood TSH estimations of normal male and female, and with special reference to cyclic variation were compared.

Urinary TSH in normal women was found to be 0.4 to $0.9 \mathrm{mu} / 100 \mathrm{ml}$, while that of normal men showed 0.1 to $0.6 \mathrm{mu} / 100 \mathrm{ml}$, showing higher $\mathrm{TSH}$ values in women. Variation by sex cycle revealed a higher TSH value during the follicle-stimulating period but became lower during the period of ovulation indicating antagonism for LH.

Although the thyroid gland during pregnancy is bound to be hyperactive in function. and morphologically as well and simultaneously TSH values would be elevated. Thus, urinary TSH $3.5 \pm 1.7 \mathrm{mu} / 100 \mathrm{ml}$ and blood TSH $45.1 \pm 3.1 \mathrm{mu} / 100 \mathrm{ml}$.

As to TSH variation by month with progress of pregnancy, it is increasingly noticeable during a period when gonadotropin lessens, that is, it increases from the third month to the 6th month and then a slight reduction occurs in the 7th month, but soon rises and usually returns to normal three to four days post-puerperium. Thus, both urinary and blood TSH show gradual increase about the time gonadotropin is lowered indicating an antagonistic attitude against gonadotropin as seen in normal cyclic period though it is parallel with estrogenic variation.

(pp. 691 705) 